\title{
Classical Modal Display Logic in the Calculus of Structures and Minimal Cut-free Deep Inference Calculi for S5
}

\author{
RAJEEV GORÉ, Computer Sciences Laboratory, The Australian National \\ University, ACT 0200 Australia. \\ E-mail: rajeev.gore@anu.edu.au
}

\begin{abstract}
ALWEN TIU, Computer Sciences Laboratory, The Australian National University and NICTA, ACT 0200 Australia.
\end{abstract}

E-mail:alwen.tiu@anu.edu.au

\begin{abstract}
We begin by showing how to faithfully encode the Classical Modal Display Logic (CMDL) of Wansing into the Calculus of Structures $(\mathrm{CoS})$ of Guglielmi. Since every CMDL calculus enjoys cut-elimination, we obtain a cut-elimination theorem for all corresponding CoS calculi. We then show how our result leads to a minimal cut-free CoS calculus for modal logic S5. No other existing CoS calculi for S5 enjoy both these properties simultaneously.
\end{abstract}

Keywords: Calculus of structures, cut-free sequent calculi, deep inference, display logic, proof theory of modal logic.

\section{Introduction}

Gentzen's sequent calculus [12] has proved useful for studying various aspects of logics like decidability, consistency, definability and even automated deduction. The main result to prove is usually cut-elimination since most other interesting properties follow from this result. Cutfree sequent calculi can be found for many logics, including non-classical logics like modal and substructural logics. But traditional sequent calculi lack the modularity inherent in Hilbert calculi: that is, extending an existing cut-free sequent calculus for a logic $L$ into a cut-free sequent calculus for a conservative extension $L_{1}$ of $L$ is rarely as straight-forward as simply adding another axiom or rule. A method that often works is to add the extra axiom, inspect the cuts that then become essential, and then replace the axiom by a new rule that absorbs these essential cuts and which is therefore as powerful as the axiom. But this is not a general solution.

Various extensions of Gentzen's sequent calculi have sought to alleviate the problem of modularity while still retaining cut-elimination. Here we briefly discuss the four main ones:

Hypersequents. The hypersequents of Pottinger [22] and Avron [1] extend the notion of a traditional sequent $\Gamma \vdash \Delta$ with semantic meaning $\models \wedge \Gamma \rightarrow \bigvee \Delta$ into a hypersequent $\Gamma_{1} \vdash \Delta_{1}\left|\Gamma_{2} \vdash \Delta_{2}\right| \cdots \mid \Gamma_{n} \vdash \Delta_{n}$ with semantic meaning $\exists i .1 \leq i \leq n \& \models \bigwedge \Gamma_{i} \rightarrow \bigvee \Delta_{i}$ Numerous logics like relevant logics and modal logics can be given a satisfactory treatment in this framework, although some care is needed to ensure cut-elimination.

Display logic. The display logic (we prefer the term display calculi) of Belnap [3] adds structural proxies for every logical connective and also adds a small collection of reversible 
'display postulates' which allow us to convert any sequent $X(Z) \vdash Y$, where structure $X$ contains an occurrence of a structure $Z$, into an equivalent sequent either of the form $Z \vdash Y^{\prime}$, or $X^{\prime} \vdash Z$, depending upon the number of negative structural connectives which govern the occurrence of $Z$. The resulting sequent is said to 'display' the occurrence of $Z$, whence the name display logic. The most remarkable feature of display calculi is a single uniform cutelimination theorem which applies whenever the rules of the display calculus obey eight easily checked conditions defined by Belnap. Numerous logics like substructural logics and modal logics can be given a uniform (cut-free) treatment in this framework too $[15,23,32]$, but as Avron points out, the cut-elimination result is not nearly as useful since it does not imply a true 'subformula property'.

Labelled sequent calculi. These calculi explicitly bring the traditional binary or ternary Kripkeframe semantics of non-classical logics into the syntax of sequents by allowing us to build sequents from labelled formulae of the form $x: A$, which informally captures that $A$ is 'true' at world $x$. Modular treatments of modal [11, 16, 21] and substructural logics [2, 31] easily follow, by utilising extra 'formulae' of the form $R(x, y)$ or $R(x, y, z)$. Such calculi can even be used for automated deduction, but the underlying Kripke conditions often become unwieldy and the need to keep the whole underlying Kripke model at all times means that these methods rarely meet the known space-complexity for the logics concerned. Moreover, this avenue is not open to us if our logic does not possess a natural Kripke-style semantics.

Calculus of structures (CoS). These calculi collapse the distinction between logical and structural connectives, work best for logics with an involutive negation, and allow rules to be applied anywhere to rewrite one structure into another. This built-in substitution principle is usually a consequence of cut-elimination in the other formalisms, so the cut rule of deep inference calculi reduces to a form of $A \wedge \neg A \rightarrow \perp$. The most surprising feature of deep inference is that it can provide cut-free calculi for logics for which it is impossible to provide a cut-free Gentzen sequent formulation [30]. Deep inference calculi have been given for some traditional modal logics [25] and also for intuitionistic logic [29]. However, direct cut-elimination proofs for $\mathrm{CoS}$ calculi have only been studied for classical logic and extensions of linear logic. The cut-elimination theorems for CoS calculi for modal and intuitionistic logics in the literature are proved indirectly via translations to other calculi. For example, the cut-elimination theorem for the deep inference calculus for modal logic $\mathbf{S 5}$ relies on the cut-elimination theorem for a hypersequent calculus for the same logic [27].

As stated previously, both hypersequents and labelled sequents allow us to give a uniform treatment of many modal logics, as long as we are willing to prove cut-elimination on an individual basis. Recent results of Brünnler [5] have shown two ways to capture many traditional modal logics uniformly, in a cut-free manner, without recourse to labels, and without recourse to indirect cut-elimination arguments. But, as far as we know, the classical modal display calculi (CMDL) of Wansing [32] captures the broadest range of modal and tense logics in a uniform and cut-free manner, particularly in the guise formulated by Kracht [20].

Here we show that deep inference can be used to give a modular treatment of all modal and tense logics from the CMDL framework. The resulting deep inference calculi are cut-free, and enjoy properties like locality, the finite choice property and even atomic contraction, but our proof of cut-elimination still relies on the general cut-elimination theorem of display calculi. 
As an application of our results we investigate three cut-free formulations of the modal logic S5 in CoS. Specifically, Stouppa [27] has recently given a cut-free CoS calculus for S5 which essentially corresponds to its Hilbert axiomatization as KT45. But it is known that S5 can also be axiomatized as KT4B and as KT5. Thus Stouppa's calculus is not minimal. We use our analysis of CMDL in $\mathrm{CoS}$ to find two cut-free $\mathrm{CoS}$ calculi, corresponding to KT4B and KT5 respectively. As far as we know, this is the first truly minimal cut-free CoS calculus for $\mathbf{S 5}$.

The article is set out as follows. We assume that the reader is familiar with either CoS or CMDL but briefly overview each in Section 2 and Section 3. Section 4 describes the formulation of CMDL in $\mathrm{CoS}$ as the calculus SMS. Section 5 shows that SMS enjoys soundness, completeness and cut-elimination. Section 8 shows how to extend SMS to capture all properly displayable 'primitive' modal and tense logics. Section 6 shows that all these extensions enjoy various important properties like locality, finite choice property, atomic contraction and cut-admissibility. Section 10 concludes with a discussion of generalizations and future work.

\section{Overview of the calculus of structures}

The calculus of structures (CoS) [17] is a particular formalism that employs deep inference rules, where rules can be applied to arbitrary subexpressions in a given logical expression. Deep inference is an old concept in proof theory, which dates back to Schütte's work [24] in the 1970s. Systems in the calculus of structures can be seen as rewrite systems. A rule scheme in $\mathrm{CoS}$ typically has the form

$$
\rho \frac{S\{T\}}{S\{R\}}
$$

where $\rho$ is the name of the rule, $R$ and $T$ are formulae (or more generally logical expressions) and $S\{\}$ is a formula-context. In general, $S\{\}$ can be an arbitrary context, but in the current article we restrict $S\{\}$ to strictly positive contexts: that is, the hole does not appear under any negation. We will work only with logics which have involutive negations, hence the negations can be pushed to the atomic level, giving a formula in negation normal form.

Basically, every valid implication $R \supset T$ can be turned into a rule in CoS as above so that the $\mathrm{CoS}$ calculus is essentially a 'right sided' calculus. The guiding principles for selecting a set of rules out of the possibly infinite choices are locality and the finite choice property of the rule as described next.

Locality means that in applying the rule in backward proof search, one does not have to inspect the entire context or the entire formula involved. The rules for modalities in sequent calculus are usually non-local in this respect, since they require that the context is of certain forms. For example, the traditional rule for introducing $\square$ into the right hand side of a sequent requires the conclusion to be of the form $\Sigma, \square \Delta \vdash \square \varphi, \Pi$, where $\Sigma$ contains no $\square$-formulae, requiring us to partition the left-hand side into two disjoint sets.

The finite choice property is reminiscent of the 'subformula property' in sequent calculus. A rule $\rho$ has the finite choice property if, for every formula $F$, there are only a finite number of instances of $\rho$ such that the conclusion of the rule matches the formula. 
There are two basic rules that are common to all classical systems in the calculus of structures $[6,17,28]$ the so-called interaction rules shown as follows, where $\bar{A}$ is the negation of $A$, and both are in negation normal form:

$$
\mathbf{i} \downarrow \frac{S\{\top\}}{S\{A \vee \bar{A}\}} \quad \mathbf{i} \uparrow \frac{S\{A \wedge \bar{A}\}}{S\{\perp\}}
$$

Notice that in classical logic, the rules correspond to the theorem $\top \supset A \vee \bar{A}$ and its contrapositive $A \wedge \bar{A} \supset \perp$. These rules are counterparts of the identity and cut rules in sequent calculus. That is, the single-sided version $\vdash p, \neg$ p, $\Sigma$ of the identity rule $\Gamma, p \vdash p, \Delta$ corresponds to accepting $\top \supset p \vee \neg p$ since the identity rule always stops backward proof search, while the single-sided premises $\vdash A, \Sigma$ and $\vdash \bar{A}, \Sigma$ of the single-sided cut rule with conclusion $\vdash \Sigma$ can instead be seen as a backward application of the $(\wedge r)$ rule to the equivalent conclusion $\vdash \Sigma, \perp$, which corresponds to accepting $A \wedge \bar{A} \supset \perp$.

The rules in CoS usually come in pairs of down-rules and up-rules which satisfy some duality property. More precisely, given a down rule $\rho \downarrow$ as shown below left, the corresponding up rule $\rho \uparrow$ is obtained by its 'contrapositive' as shown below right:

$$
\rho \downarrow \frac{S\{T\}}{S\{R\}} \quad \rho \uparrow \frac{S\{\bar{R}\}}{S\{\bar{T}\}}
$$

The down rules are rules which have the locality and finite choice properties, and are the ones we wish to retain. The up-rules, such as the $\mathbf{i} \uparrow$ rule earlier, are needed to simulate the cut rules (Section 4) and are admissible for provability. The up-rules usually do not satisfy the finite choice property. For example, the $\mathbf{i} \uparrow$ rule is non-finite, since we can instantiate $A$ with any formula and still have the conclusion of the rule instance match $\perp$. The cut-elimination theorem in $\mathrm{CoS}$ is therefore the ability to eliminate the up-rules.

\section{Classical Modal Display Logic}

Display Logic is a generalized sequent calculus framework due to Nuel Belnap [3]. The following characteristics are fundamental to all display calculi $[14,15]$. For modal logic, we follow the notation of Kracht [20] rather than Belnap or Wansing [32] since we rely on results from Kracht, except that we use slightly different notation for the two past-time modalities, and we make a small but insignificant change to their introduction rules.

Structural connectives. Display calculi utilize one extra structural connective for every pair of dual or residual logical connectives. The structural connective is overloaded just as Gentzen overloaded the structural connective comma to mean conjunction when appearing on the left of turnstile and disjunction when appearing on the right of turnstile. Depending upon the monotonic or anti-monotonic properties of the dual/residual logical connectives it captures, the new structural connective has 'positive' positions and/or 'negative' positions.

CMDL structures $X$ are built from CMDL formulae $F$ and CMDL structural constant I according to the grammar below:

$$
\begin{aligned}
& F::=\top|\perp| p|F \vee F| F \wedge F|\neg F| \boldsymbol{\square} F|\diamond F| \square F \mid \diamond F \\
& X::=F|\mathbf{I}| * X|\bullet X| X \circ X
\end{aligned}
$$




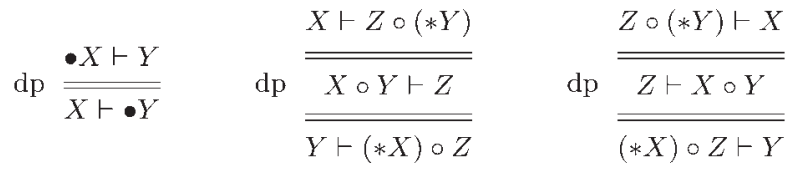

$$
\begin{aligned}
& \mathrm{d} p \frac{* X \vdash Y}{* Y \vdash X} \quad \mathrm{dp} \frac{* * X \vdash Y}{X \vdash Y} \quad \mathrm{dp} \frac{Y \vdash * * X}{Y \vdash X} \quad \mathrm{dp} \frac{Y \vdash * X}{\overline{X \vdash * Y}}
\end{aligned}
$$

Figure 1. Some display postulates in CMDL

$$
\begin{aligned}
& \text { Id } p \vdash p \quad \operatorname{cut} \frac{X \vdash F \quad F \vdash Y}{X \vdash Y} \quad \neg l \frac{* F \vdash Z}{\neg F \vdash Z} \quad \neg r \frac{Z \vdash * F}{Z \vdash \neg F} \\
& \wedge \frac{F \circ G \vdash Z}{F \wedge G \vdash Z} \quad \wedge r \frac{X \vdash F \quad Y \vdash G}{X \circ Y \vdash F \wedge G} \\
& \bullet \frac{\bullet F \vdash Z}{\bullet F \vdash Z} \quad r \frac{Z \vdash F}{\bullet Z \vdash F} \quad \square l \frac{F \vdash Z}{\square F \vdash \bullet Z} \quad \square r \frac{Z \vdash \bullet F}{Z \vdash \square F} \\
& \diamond l \frac{* \bullet * F \vdash Z}{\diamond F \vdash Z} \quad \diamond r \frac{Z \vdash F}{* \bullet * Z \vdash \vartheta F} \quad \boldsymbol{\square} \frac{F \vdash Z}{\square F \vdash * * Z} \quad \mathbf{\square} r \frac{Z \vdash * \bullet * F}{Z \vdash \boldsymbol{\square} F}
\end{aligned}
$$

Figure 2. Some logical rules in CMDL

Structure $Z$ occurs negatively in $* Z$, and occurs positively in each of $\bullet Z$ and $(Y \circ Z)$ and $(Z \circ Y)$. A proper structure is a structure which is not a formula.

Display Sequents. A display sequent $X \vdash Y$ consists of two arbitrary structures $X$ and $Y$ built up from formulae using the formation rules for these extra structural connectives. Substructure $Z$ is an antecedent [succedent] part of $X \vdash Y$ if it occurs positively [negatively] in $X$ or negatively [positively] in $Y$.

Display postulates and display property. Display calculi also require a basic collection of reversible sequent rules called the 'display postulates' which allow us to dis-assemble and re-assemble arbitrary CMDL structures. The defining property is that for proper structure $Z$ occurring in a sequent $X \vdash Y$, there is a display-equivalent sequent $Z \vdash Y^{\prime}$ or $X^{\prime} \vdash Z$ depending only upon whether $Z$ is an antecedent part or succedent part of the sequent, respectively. The resulting sequent is said to display $Z$, whence the name display logic.

CMDL contains the display postulates given in Figure 1. The following theorem then holds.

THEOREM 1 (Display property)

For every antecedent [succedent] part $\mathrm{Z}$ of sequent $\mathrm{X} \vdash \mathrm{Y}$, there is a sequent $Z \vdash Y^{\prime}\left[X^{\prime} \vdash Z\right.$ ] which is obtainable by the application of the display postulates only. Structure $Z$ is said to be displayed in the resulting sequent.

Logical rules maintaining display property. Rules for introducing each logical connective into the left or right of turnstile maintain the display property by introducing the new connective into a formula which is either the whole of the left-hand side or the whole of the right hand side of a sequent. We give some logical rules from CMDL in Figure 2 as illustrations. Notice that the structure $\circ$ is a proxy for a de Morgan dual pair $(\wedge, \vee)$, but the structure $\bullet$ is a proxy for a residuated pair $(\bullet, \square)$, and the structure $*$ is a proxy for $\neg$. Notice also that the cut rule captures only the transitivity of the turnstile. All substitutional aspects of cut are taken care of by the display property. 


$$
\begin{gathered}
\text { Coml } \frac{Y \circ X \vdash Z}{X \circ Y \vdash Z} \quad \text { Comr } \frac{Z \vdash Y \circ X}{Z \vdash X \circ Y} \quad \text { Assl } \frac{(X \circ Y) \circ Z \vdash W}{X \circ(Y \circ Z) \vdash W} \\
\text { Assr } \frac{W \vdash X \circ(Y \circ Z)}{W \vdash(X \circ Y) \circ Z} \quad \text { W1 } \frac{Y \vdash Z}{X \circ Y \vdash Z} \quad \text { Wr } \frac{Z \vdash X}{Z \vdash X \circ Y} \\
\text { Cl } \frac{X \circ X \vdash Z}{X \vdash Z} \quad \operatorname{Cr} \frac{Z \vdash X \circ X}{Z \vdash X}
\end{gathered}
$$

Figure 3. Some structural rules in CMDL

A good test of sanity for the rules is the following lemma.

\section{LEMMA 2}

For every formula $F$, the sequent $F \vdash F$ is cut-free derivable in CMDL.

Modularity via further structural rules. Further structural rules like associativity, commutativity, contraction and weakening can be added separately in a modular fashion.

CMDL has a classical basis, so we have commutativity, associativity, weakening and contraction as shown in Figure 3, amongst others which we have omitted for brevity (see [20] for details).

Cut elimination. The most amazing feature of all display calculi is that one single cutelimination procedure works for all display calculi whose rules obey eight easily checked conditions stipulated by Belnap and machine-checked by Dawson and Goré [8,9]. There is also a general methodology for constructing a display calculus for many different logics [14].

All rules of CMDL obey these eight conditions, hence we have the following theorem.

THEOREM 3 (Cut elimination)

If a sequent $\mathrm{X} \vdash \mathrm{Y}$ is derivable in $\mathrm{CMDL}$ then it is derivable without the cut rule.

\section{Modal tense logic in $\mathrm{CoS}$}

In this section we present a formulation of CMDL in the calculus of structures. We will work only with formulae in which negations appear only at the atomic level, since negation in CMDL is involutive and can be pushed to the atomic level. Note also that there is no connective for implication so implications have to be translated away with $A \supset B$ considered an abbreviation of $\neg A \vee B$. The logical expressions in CoS are also called structures, and are generated by the following grammar, where $a$ denotes a propositional variable and $\bar{a}$ denotes its negation:

$$
R::=\mathbf{t}|\mathbf{f}| a|\bar{a}|[R, R]|(R, R)| \mathbf{\square} R|\diamond R| \square R \mid \diamond R
$$

The CoS constants $\mathbf{t}$ and $\mathbf{f}$ correspond to the constants $T$ and $\perp$. The CoS structural connective [ ] and ( ) denote disjunction and conjunction, respectively, with comma once again overloaded depending upon its context. The modalities retain their meaning in CoS structures. Note that the use of bracketing notation to present structures is just for typographical purpose to make the proof figures more readable. The dual of a structure $R$, denoted by $\bar{R}$, is the structure obtained by interchanging each structural connective with 


$$
\begin{aligned}
& \mathbf{i} \downarrow \frac{S\{\mathbf{t}\}}{S[R, \bar{R}]} \quad \mathbf{i} \uparrow \frac{S(R, \bar{R})}{S\{\mathbf{f}\}} \quad \mathrm{s} \frac{S([R, T], U)}{S[R,(T, U)]} \\
& \mathbf{c} \downarrow \frac{S[R, R]}{S\{R\}} \quad \mathbf{c} \uparrow \frac{S\{R\}}{S(R, R)} \quad \mathbf{w} \downarrow \frac{S\{\mathbf{f}\}}{S\{R\}} \quad \mathbf{w} \uparrow \frac{S\{R\}}{S\{\mathbf{t}\}} \\
& \mathbf{k f} \downarrow \frac{S\{\square[R, T]\}}{S[\diamond R, \square T]} \quad \mathbf{k f} \uparrow \frac{S(\square R, \diamond T)}{S\{\diamond(R, T)\}} \quad \mathbf{k p} \downarrow \frac{S\{\boldsymbol{\square}[R, T]\}}{S[R, \boldsymbol{\square} T]} \quad \operatorname{kp} \uparrow \frac{S(\boldsymbol{\square} R, \diamond T)}{S\{(R, T)\}} \\
& \operatorname{rpf} \downarrow \frac{S\{\square[\mathbf{\square} R, T]\}}{S[R, \square T]} \quad \operatorname{rpf} \uparrow \frac{S(R, \diamond T)}{S\{\diamond(\diamond R, T)\}} \quad \operatorname{rpp} \downarrow \frac{S\{\mathbf{\square}[\square R, T]\}}{S[R, \mathbf{\square} T]} \quad \operatorname{rpp} \uparrow \frac{S(R, \diamond T)}{S\{\diamond(\diamond R, T)\}}
\end{aligned}
$$

Figure 4. System SMS

its dual and each atom with its dual. A structure context is a structure with a hole. Structure contexts are denoted with $S\{\}$. We omit the outermost braces if they would immediately enclose brackets or parentheses, writing $S[R, \mathbf{f}]$ for $S\{[R, \mathbf{f}]\}$ for example. Structures are considered modulo the equational theory as follows:

$\begin{array}{lll}\text { Units: } & {[R, \mathbf{f}]=R} & (R, \mathbf{t})=R \\ & \mathbf{t}=\square \mathbf{t}=\mathbf{\square} \mathbf{t}=[\mathbf{t}, \mathbf{t}] & \mathbf{f}=\diamond \mathbf{f}=\diamond \mathbf{f}=(\mathbf{f}, \mathbf{f}) \\ \text { Associativity: } & {[R,[T, U]]=[[R, T], U]} & (R,(T, U))=((R, T), U) \\ \text { Commutativity: } & {[R, T]=[T, R]} & (R, T)=(T, R) \\ \text { Congruence: } & \text { If } R=T \text { then } S\{R\}=S\{T\} . & \end{array}$

\section{DeFinITION 4}

The rules for system SMS are given in Figure 4. System MS is obtained by removing all the up-rules from SMS, leaving MS as the set $\{\mathbf{i} \downarrow, \mathbf{s}, \mathbf{c} \downarrow, \mathbf{w} \downarrow, \mathbf{k f} \downarrow, \mathbf{k p} \downarrow, \mathbf{r p f} \downarrow, \mathbf{r p p} \downarrow\}$. The subsystem $\{\mathbf{i} \downarrow, \mathbf{s}, \mathbf{c} \downarrow, \mathbf{w} \downarrow\}$ is called $\mathbf{K S g}$ and corresponds to propositional classical logic [6, 7] (when restricted to propositional structures, naturally). A derivation in a logical system $\mathcal{S}$ in the calculus of structures is a sequence of instances of rules from $\mathcal{S}$. Derivations are denoted with $\Pi$ or $\Delta$. The graphical notation

$$
\stackrel{R}{\Delta \|_{T}^{R} \mathcal{S}}
$$

denotes a derivation $\Delta$ from $R$ to $T$ in a system $\mathcal{S}$. A proof in $\mathcal{S}$ of a structure $R$ is a derivation in $\mathcal{S}$ from $\mathbf{t}$ to $R$. We sometimes omit the derivation name (e.g. $\Delta$ in the above derivation) or system name from the graphical representation of a derivation when they are not relevant or can be inferred from the context. We also use the notation $\Pi \vdash_{\mathcal{S}} R$ to denote that $\Pi$ is a proof of $R$ in system $\mathcal{S}$, and $\vdash_{\mathcal{S}} R$ to denote that $R$ is provable in system $\mathcal{S}$. 
The cut rule in (one-sided) sequent calculus can be derived in SMS as follows (modulo structural equivalence):

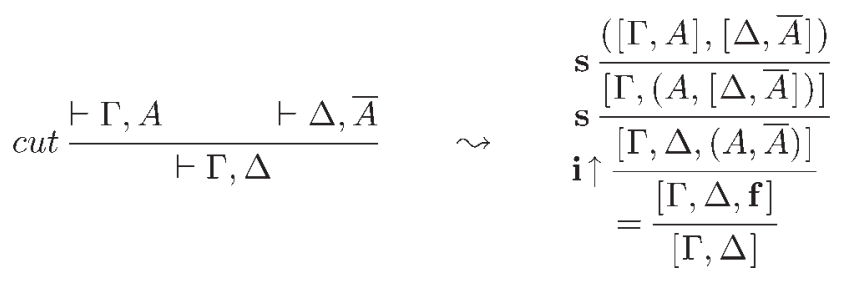

DEFINITION 5

Two logical systems $\mathcal{S}_{1}$ and $\mathcal{S}_{2}$ are equivalent if they prove the same set of structures: that is, for every $R$ we have $\vdash_{\mathcal{S}_{1}} R$ if and only if $\vdash_{\mathcal{S}_{2}} R$.

Definition 6

The dual of a rule scheme

$$
\rho \frac{S\{T\}}{S\{R\}}
$$

is obtained by exchanging the premise and the conclusion of $\rho$, and exchanging every structural connective in $R$ and $T$ with its dual. For instance, the rules $\rho_{1}$ and $\rho_{2}$ are dual to each other, as are $\rho_{3}$ and $\rho_{4}$ :

$$
\rho_{1} \frac{S[\square R, \square T]}{S\{\square[R, T]\}} \quad \rho_{2} \frac{S\{\diamond(R, T)\}}{S(\diamond R, \diamond T)} \quad \rho_{3} \frac{S[\mathbf{\square}, \boldsymbol{\square} T]}{S\{\boldsymbol{\square}[R, T]\}} \quad \rho_{4} \frac{S\{\diamond(R, T)\}}{S(\diamond R, \diamond T)}
$$

A system $\mathcal{S}$ is symmetrically closed if for every rule $\rho_{1} \in \mathcal{S}$, its dual is also in $\mathcal{S}$. The symmetric closure of a system $\mathcal{S}$ is a system obtained by adding to $\mathcal{S}$ the duals of its rules.

For example, system SMS is symmetrically closed, and it is obtained by applying symmetric closure to MS. The cut-elimination theorem for SMS, as we shall see, essentially says that the symmetric closure SMS of system MS is no more powerful than the system MS itself, in terms of provability.

\section{Soundness, completeness and cut-elimination for SMS}

We assume familiarity with display calculi, particularly the CMDL of Wansing [32] as studied by Kracht [20] and Goré [13].

We make use of some derived display postulates, stated in the following lemma. Its proof is straightforward by applications of basic display postulates and structural rules.

LEMMA 7

The following rules are cut-free derivable in CMDL:

$$
\begin{array}{ccc}
r_{1} \frac{(\bullet X) \circ(\bullet Y) \vdash Z}{\bullet(X \circ Y) \vdash Z} \quad r_{2} \frac{X \vdash(\bullet Y) \circ(\bullet Z)}{X \vdash \bullet(Y \circ Z)} & r_{3} \frac{X \vdash * \bullet * Y}{* \bullet X \vdash Y} \quad r_{4} \frac{* \bullet X \vdash Y}{X \vdash * \bullet * Y} \\
r_{5} \frac{(* \bullet * X) \circ(* \bullet * Y) \vdash Z}{* \bullet *(X \circ Y) \vdash Z} \quad r_{6} \frac{X \vdash(* \bullet * Y) \circ(* \bullet * Z)}{X \vdash * \bullet *(Y \circ Z)}
\end{array}
$$


DEFINITION 8

Translations between CoS structures and CMDL formulae. We consider only CMDL formulae which are in negation normal form: that is, all negations are pushed to the atomic level. Given a CMDL formula $F$, we denote with $\bar{F}$ its negation which is in negation normal form. Given a CoS structure $R$, we denote with $\underline{R}_{\mathrm{F}}$ the CMDL formula obtained from $R$ by replacing t with $\top$, f with $\perp$, and replacing substructures $\left[R_{1}, R_{2}\right]$ with $R_{1_{\mathrm{F}}} \vee R_{2} \mathrm{~F}$ and $\left(R_{1}, R_{2}\right)$ with $\underline{R_{1}} \underline{\mathrm{F}} \wedge{\underline{R_{2}}}_{\mathrm{F}}$. Conversely, given a CMDL formula $F$, we define a $\overline{\operatorname{CoS}}$ structure $\underline{F}_{\mathrm{S}}$ as follows:

$$
\begin{array}{ccc}
\underline{I}_{s}=\mathbf{t} \quad \underline{\perp}_{s}=\mathbf{f} & \underline{a}_{s}=a \quad \neg a_{s}=\bar{a} \\
\underline{A \vee B_{s}}=\left[\underline{A}_{s}, \underline{B}_{s}\right] & \underline{A \wedge B_{s}}=\left(\underline{A}_{s}, \underline{B}_{s}\right) \\
A \supset B_{s}=\left[\underline{\bar{A}}_{s}, \underline{B}_{s}\right] & \neg \underline{A}_{s}=\underline{\bar{A}}_{s} \\
\square \underline{A}_{s}=\square \underline{A}_{s} \quad \underline{A}_{s}=\diamond \underline{A}_{s} & \square A_{s}=\mathbf{\square} \underline{A}_{s} \quad \stackrel{\bullet}{A_{s}}=\diamond \underline{A}_{s} .
\end{array}
$$

To translate CMDL sequents to CoS structures, we first translate their formula counterparts. That is, we first use a translation from CMDL sequents to CMDL formulae as follows [32] where $P$ is a CMDL formula:

$$
\begin{array}{cccccc}
\multicolumn{7}{c}{\tau(X \vdash Y)=\tau_{1}(X) \supset \tau_{2}(Y)} \\
\tau_{1}(P) & = & P & \tau_{2}(P) & = & P \\
\tau_{1}(\mathbf{I}) & = & \top & \tau_{2}(\mathbf{I}) & = & \perp \\
\tau_{1}(* X) & = & \neg \tau_{2}(X) & \tau_{2}(* X) & = & \neg \tau_{1}(X) \\
\tau_{1}(X \circ Y) & = & \tau_{1}(X) \wedge \tau_{1}(Y) & \tau_{2}(X \circ Y) & = & \tau_{2}(X) \vee \tau_{2}(Y) \\
\tau_{1}(\bullet X) & = & \diamond \tau_{1}(X) & \tau_{2}(\bullet) & = & \square \tau_{2}(X)
\end{array}
$$

The translation from a CMDL sequent to a $\operatorname{CoS}$ structure is then defined as

$$
\underline{X \vdash Y} \underline{\mathrm{ss}}=\underline{\tau(X \vdash Y)} \mathrm{s}
$$

The following lemma just confirms that the two translations are inverses of each other, and follows from their definitions.

LEMMA 9

For every CoS structure $R$, we have $\underline{\underline{R}}_{\mathrm{F}_{\mathrm{s}}}=R$.

LEMMA 10 (Soundness)

If $\operatorname{CoS}$ structure $R$ is provable in SMS then CMDL sequent $\mathbf{I} \vdash \underline{R}_{\mathrm{F}}$ is provable in CMDL.

Proof. Let $\Pi$ be a proof of $R$ in SMS. We prove the lemma by induction on the length of $\Pi$.

Suppose $R$ is of the form $S\left\{T^{\prime}\right\}$ and $\Pi$ ends with a rule $\rho$ so that $\Pi$ is of the form shown below left. The CMDL proof of $R$, i.e. $S\left\{T^{\prime}\right\}$, is constructed by applying the cut rule shown below right.

$$
\begin{aligned}
& \underset{S\left\{R^{\prime}\right\}}{\Pi^{\prime} \|} \quad \operatorname{cut} \frac{\mathbf{I} \vdash S\left\{R^{\prime}\right\}_{\mathbf{F}} \quad S\left\{R^{\prime}\right\}_{\mathrm{F}} \vdash \underline{S\left\{T^{\prime}\right\}_{\mathrm{F}}}}{\mathbf{I} \vdash \underline{S\left\{T^{\prime}\right\}_{\mathrm{F}}}} \\
& \rho \frac{S\left\{R^{\prime}\right\}}{S\left\{T^{\prime}\right\}}
\end{aligned}
$$

The left premise of the cut is provable in CMDL by induction hypothesis. To prove the right premise, it is sufficient to show that $\underline{R}_{\mathrm{F}}^{\prime} \vdash \underline{T}_{\mathrm{F}}^{\prime}$ is provable, which can be done by 

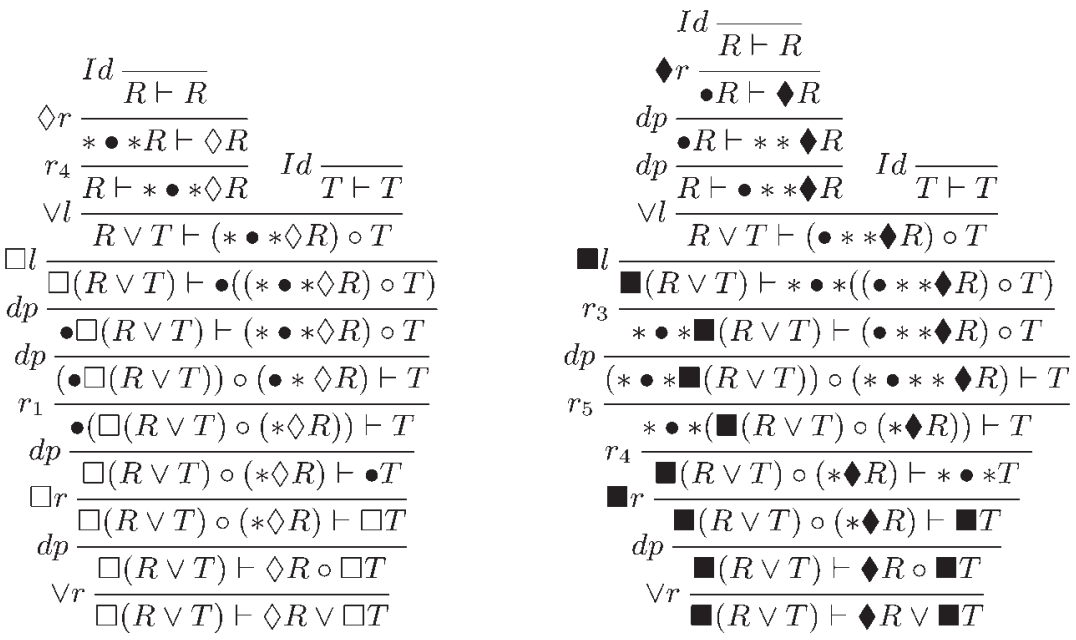

FIGURE 5. Soundness of $\mathbf{k f} \downarrow$ and $\mathbf{k p} \downarrow$

a straightforward induction on the size of the context $S\{\}$. Thus, it is enough to show that each rule in SMS corresponds to a valid implication in CMDL.

Below we show the CMDL proofs for several $\mathrm{CoS}$ rule schemes involving modalities. Moreover, we show only the derivation of the down-rules; the derivations of the up-rules can be obtained using a symmetric argument, since the implications defining the rules are the contrapositives of the implications defining the down-rules. The translation function is omitted in the proofs below to simplify the presentation. Derivations for the rule schemes for $\mathbf{k f} \downarrow$ and $\mathbf{k p} \downarrow$

$$
\mathbf{k f} \downarrow \frac{S\{\square[R, T]\}}{S[\diamond R, \square T]} \quad \mathbf{k p} \downarrow \frac{S\{\mathbf{\square}[R, T]\}}{S[\diamond R, \square T]}
$$

are shown in Figure 5. Derivations for

$$
\text { rpf } \downarrow \frac{S\{\square[\mathbf{\square}, T]\}}{S[R, \square T]} \quad \operatorname{rpp} \downarrow \frac{S\{\mathbf{\square}[\square R, T]\}}{S[R, \boldsymbol{\square} T]}
$$

are given in Figure 6.

LEMMA 11 (Necessitation)

If $R$ is provable in SMS or MS then $\square R$ and $\square R$ are provable in SMS or MS respectively.

Proof. Let $\Pi$ be a $\operatorname{CoS}$ proof of $R$, and let $\Pi^{\prime}$ be a $\operatorname{CoS}$ derivation obtained from $\Pi$ by adding a $\square$ to every structure $T$ in $\Pi$. The CoS proof of $\square R$ is then:

$$
\begin{gathered}
=\frac{\mathbf{t}}{\square \mathbf{t}} \\
\Pi^{\prime} \| \\
\square R
\end{gathered}
$$

To get a CoS proof of $\boldsymbol{\square}$, apply the same construction but with $\boldsymbol{\square}$ replacing $\square$.

LEMMA 12

If $X \vdash Y$ is cut-free provable in CMDL then $\underline{X \vdash} \underline{S S}_{\mathrm{SS}}$ is provable in MS. 

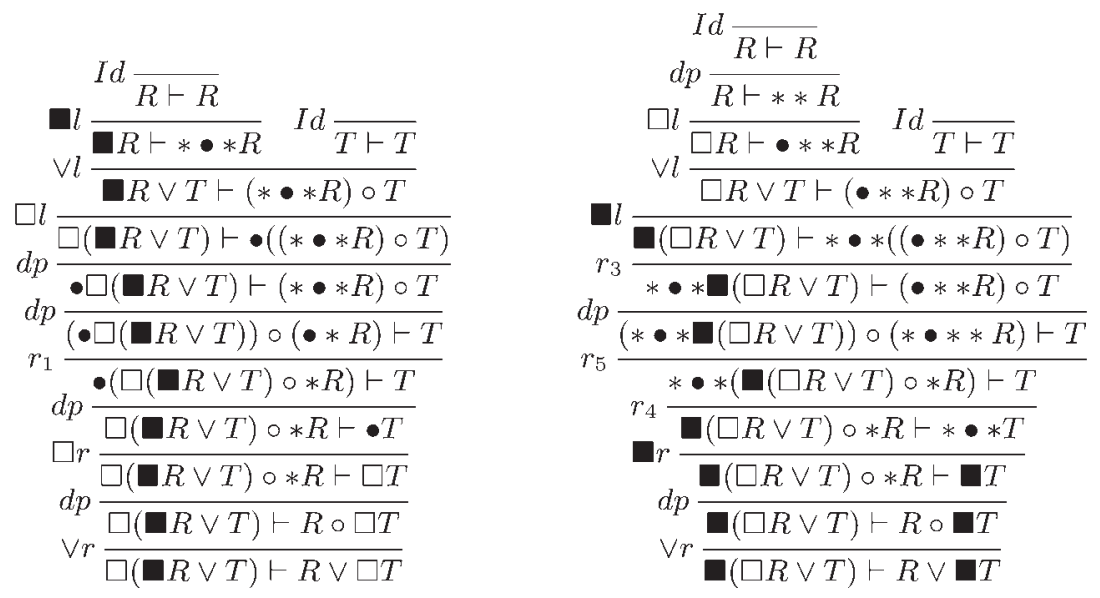

Figure 6. Soundness of $\mathbf{r p f} \downarrow$ and $\mathbf{r p p} \downarrow$

Proof. Let $\Pi$ be a CMDL proof of $X \vdash Y$. The lemma is proved by induction on the height of $\Pi$. We distinguish several cases based on the last rule in $\Pi$. These cases fall under the more general categories of the identity rule, structural rules and logical rules. We omit some trivial cases like the identity rule, the structural rules for associativity of o, commutativity of $\circ$, and the rules for the unit $\mathbf{I}$. The cases shown are representative of the kind of transformations needed to simulate CMDL proofs inside $\mathrm{CoS}$. We omit the translation function $\underline{\underline{\mathrm{S}}}$ to simplify the presentation.

\section{Structural rules:}

(1) Suppose $\Pi$ ends with the rule instance of $d p$ as shown below left. Applying the $\tau$ function to $\bullet X \vdash Y^{\prime}$ and $X \vdash \bullet Y^{\prime}$ gives the CMDL formulae $\diamond A \supset B$ and $A \supset \square B$ for some $A$ and $B$.

By the induction hypothesis, there is a proof $\Delta$ in MS of the $\operatorname{CoS}$ structure $[\mathbf{\square} \bar{A}, B]$ since this is $A \supset B_{\mathrm{S}}$. We need to show that there is a proof of $[\bar{A}, \square B]$ i.e. $\underline{A \supset \square B_{\mathrm{S}}}$ in MS. By the necessitation Lemma11, there is a proof $\Delta^{\prime}$ of $\square[\square \bar{A}, B]$. The proof of $[\bar{A}, \square B]$ is therefore constructed as below right:
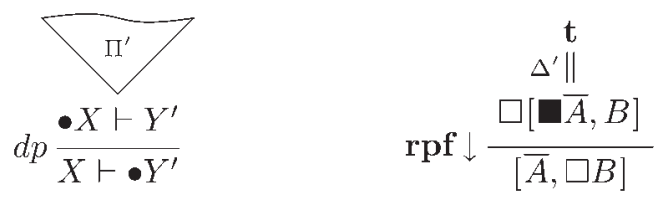

(2) Suppose $\Pi$ is as shown below left. This case is similar to the previous one, but with the roles of $\triangleleft \supset \mathrm{B}$ and $A \supset \square B$ reversed and with $\mathbf{r p p} \downarrow$ replacing $\mathbf{r p f} \downarrow$.
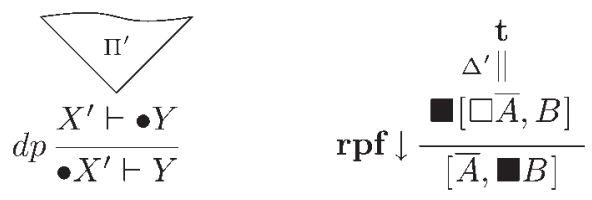


\section{Logical rules:}

(1) Suppose $\Pi$ with conclusion $X^{\prime} \circ Y^{\prime} \vdash P \wedge Q$ is as shown below left. The CMDL sequents $X^{\prime} \vdash P$ and $Y^{\prime} \vdash Q$ and $X^{\prime} \circ Y^{\prime} \vdash P \wedge Q$ translate respectively to the $\operatorname{CoS}$ structures $[R, T]$ and $[V, U]$ and $[[R, V],(T, U)]$ for some $R, T, V$ and $U$. By the induction hypotheses, we have derivations $\Delta_{1}$ and $\Delta_{2}$ such that $\Delta_{1} \vdash[R, T]$ and $\Delta_{2} \vdash[V, U]$. The proof for $[[R, V],(T, U)]$ is constructed as shown below right where $\Delta_{2}^{\prime}$ is obtained by nesting $\Delta_{2}$ in the hole in the context $[R,(T,\{\})]$ :

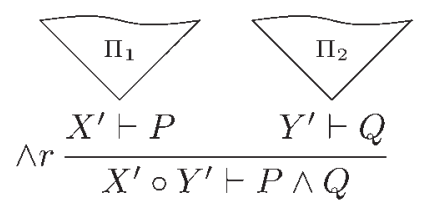

$$
\begin{aligned}
& \Delta_{1} \\
& =\frac{[R, T]}{[R,(T, \mathbf{t})]} \\
& \Delta_{2}^{\prime} \| \\
& =\frac{\mathbf{s} \frac{[R,(T,[V, U])]}{[R,[V,(T, U)]]}}{[[R, V],(T, U)]}
\end{aligned}
$$

The case with the left introduction rule for $\vee$ and $\supset$ can be treated similarly. The cases involving the right introduction for $\vee$, the right introduction for $\supset$ and the left introduction for $\wedge$ are trivial since in these cases the premise and the conclusion of the introduction rules are translated to equivalent $\mathrm{CoS}$ structures.

(2) The cases where $\Pi$ ends with one of the modal rules $\square r, \diamond l, \boldsymbol{\square} r$ and $\diamond l$ are trivial, since in these cases the premise and the conclusion of the last rule translate to equivalent $\mathrm{CoS}$ structures. Our 'insignificant' changes to the CMDL introduction rules of Kracht are particularly useful here.

(3) Suppose $\Pi$ is as shown below left. The premise and the conclusion in the last rule translate to $[R, T]$ and $[\diamond R, \square T]$, respectively. By the induction hypothesis and Lemma 11 , there is a proof $\Delta$ of $\square[R, T]$. The proof of $[\diamond R, \square T]$ is constructed as below right:

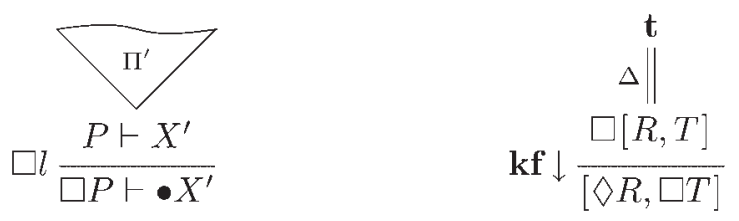

(4) Suppose $\Pi$ ends with $\diamond r$, i.e.

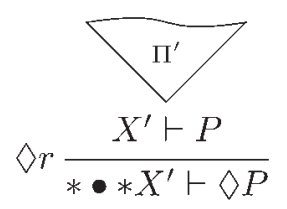


In this case, the premise and the conclusion sequents translate to the CoS structures $[R, T]$ and $[\square R, \diamond T]$ for some $R$ and $T$. Notice that these are the same structure schemes to those in the $\square l$ case, except with $R$ and $T$ interchanged. We therefore apply the same construction to get a proof of $[\square R, \diamond T]$.

(5) Suppose $\Pi$ ends with $\diamond r$ as shown below left: The premise and the conclusion of the last rule translate to structures $[R, T]$ and $[\square R, \diamond T]$. The proof for $[\square R, \diamond T]$ is then constructed from the induction hypothesis and the necessitation Lemma 11 (which gives $\Delta$ ) as shown below right:

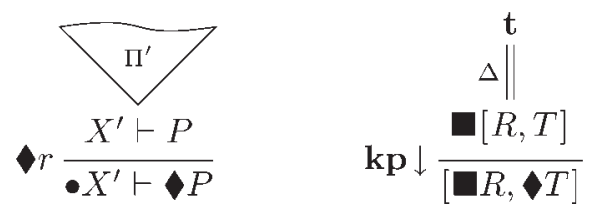

(6) If $\Pi$ ends with $\boldsymbol{\square} l$ then follow the same construction as in the case with $\diamond r$.

THEOREM 13

The following statements are equivalent, for any CoS structure $R$.

(1) $R$ is provable in SMS.

(3) $\underline{R}_{\mathrm{F}}$ is cut-free provable in CMDL.
(2) $\underline{R}_{\mathrm{F}}$ is provable in CMDL.

(4) $R$ is provable in MS.

Proof. Lemma 10 gives us that (1) implies (2). The cut-elimination result, Theorem 3, for CMDL gives us the equivalence between (2) and (3). Lemma 12 shows that (3) implies (4). And since MS is a subsystem of SMS, we have (4) implies (1).

Conservativity theorem. We now consider the question of whether MS is a conservative extension of basic modal logic K. Formalizations of $\mathbf{K}$ and several of its extensions in $\mathrm{CoS}$ have been studied in $[25,26]$. In particular, the basic modal logic $\mathbf{K}$ is shown to correspond to the system KSg $\cup\{\mathbf{k f} \downarrow\}$.

DEFINITION 14

System KSgk is KSg $\cup\{\mathbf{k f} \downarrow\}$ and SKSgk is the symmetric closure of KSgk.

THEOREM 15

Systems KSgk and SKSgk are equivalent.

Proof. See [26].

Definition 16

A modal structure/formula is a structure/formula with no occurrences of $\mathbf{\square}$ and

Theorem 17 (Conservativity)

A modal structure $R$ is provable in MS if and only if $R$ is provable in KSgk. 
Proof. Let us denote with Kt the (theorems of the) Hilbert style formulation of tense logic. It is well known that $\mathbf{K t}$ is a conservative extension of modal logic $\mathbf{K}$, but the following semantic argument proves this claim.

We note that Kt is determined by the class of all Kripke models with bi-directional frames $\left\langle W, R_{1}, R_{2}\right\rangle$ where $R_{1}=R_{2}^{-1}$, while $\mathbf{K}$ is determined by exactly the same class of frames when viewed as just $\left\langle W, R_{1}\right\rangle$. So every modal formula which has a $\mathbf{K}$-model has exactly the same Kt-model. Therefore, every modal formula which is provable in $\mathbf{K t}$ is provable in $\mathbf{K}$.

We use this fact to prove the conservativity property of MS, as illustrated in the following diagram:

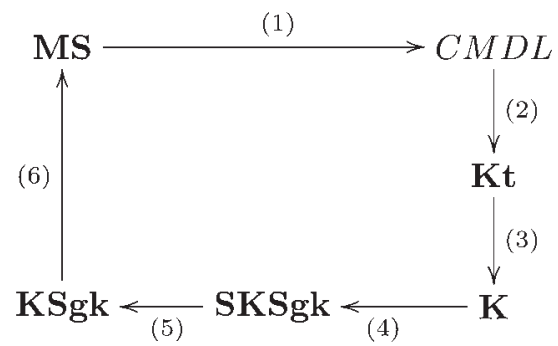

Each arrow corresponds to an implication: (1) is justified by Theorem 13, (2) by the result of Wansing [32], (3) is as described by the semantic argument above, (4) and (5) are the results of Stouppa [26] and (6) is trivial since KSgk is a subsystem of MS.

\section{Local rules}

One of the features of the calculus of structures is that the inference rules can be made local: that is, in applying the rules backward, we need to check only a bounded amount of information about the structures involved. The interaction rule is non-local, since in order to apply the rule, we need to check that two structures are dual to each other, which requires inspecting these structures completely. Similarly, the contraction rule is also non-local, since in this case we have to check that two structures are syntactically equal.

The above notion of locality has become quite standard across formulations of different logical systems in CoS: classical logic [6], linear logic [17, 28], modal logic [26] and intuitionistic and intermediate logics [29]. We show that the formulation of tense logic in CoS is not an exception. The techniques and rules shown in the following, in particular the ones that concern modalities, are essentially those of Stouppa [26], with a very straightforward extension to cover the tense modalities. This is because the reduction of the rules to their local counterparts is independent of the residuation rules, i.e. the rules $\mathbf{r p f} \downarrow, \mathbf{r p p} \downarrow$ and their duals.

Definition 18

A rule $\rho$ is derivable in a system $\mathcal{S}$ if for every instance of $\rho$, there is a derivation from the premise of $\rho$ to its conclusion in $\mathcal{S}$.

Atomic interaction rules. With the help of the switch rule $\mathbf{s}$, the modal rules $\mathbf{k f} \downarrow$, $\mathbf{k p} \downarrow$, and their duals, we can show that the general interaction rules $\mathbf{i} \downarrow$ and $\mathbf{i} \uparrow$ can be replaced by their atomic counterparts shown below:

$$
\mathbf{a i} \downarrow \frac{S\{\mathbf{t}\}}{S[a, \bar{a}]} \quad \mathbf{a i} \uparrow \frac{S(a, \bar{a})}{S\{\mathbf{f}\}}
$$


LEMMA 19

The rule $\mathbf{i} \downarrow$ is derivable in $\{\mathbf{s}, \mathbf{k f} \downarrow, \mathbf{k p} \downarrow, \mathbf{a i} \downarrow\}$. Dually, the rule $\mathbf{i} \uparrow$ is derivable in $\{\mathbf{s}, \mathbf{k f} \uparrow$, $\mathbf{k p} \uparrow, \mathbf{a i} \uparrow\}$.

Proof. See [26].

Local weakening. The general weakening rules can be reduced to more restricted forms, which are local. These restricted weakening rules are as below where $a$ denotes an atomic formula:

$$
\begin{array}{lll}
\operatorname{aw} \uparrow \frac{S\{a\}}{S\{\mathbf{t}\}} & \text { uwf } \uparrow \frac{S\{\diamond \mathbf{t}\}}{S\{\mathbf{t}\}} & \operatorname{uwp} \uparrow \frac{S\{\diamond \mathbf{t}\}}{S\{\mathbf{t}\}} \\
\operatorname{aw} \downarrow \frac{S\{\mathbf{f}\}}{S\{a\}} & \text { uwf } \downarrow \frac{S\{\mathbf{f}\}}{S\{\mathbf{f}\}} & \operatorname{uwp} \downarrow \frac{S\{\mathbf{f}\}}{S\{\square \mathbf{f}\}}
\end{array}
$$

Lemma 20

The rule $\mathbf{w} \downarrow$ is derivable in $\{\mathbf{a w} \downarrow, \mathbf{u w f} \downarrow$, uwp $\downarrow]$. The rule $\mathbf{w} \uparrow$ is derivable in \{ $\mathbf{a w} \uparrow$, uwf $\uparrow$, uwp $\uparrow\}$.

Proof. See [26].

Atomic contraction. We now show that the contraction and the co-contraction rules can be restricted to their atomic forms shown as follows:

$$
\text { ac } \downarrow \frac{S[a, a]}{S\{a\}} \quad \text { ac } \uparrow \frac{S\{a\}}{S(a, a)}
$$

provided we add the so-called medial and co-medial rules:

$$
\begin{array}{ccc}
\mathbf{m} \frac{S[(R, U),(T, V)]}{S([R, T],[U, V])} & \mathbf{m} \square \downarrow \frac{S[\square R, \square T]}{S\{\square[R, T]\}} & \mathbf{m} \square \uparrow \frac{S\{\diamond(R, T)\}}{S(\diamond R, \diamond T)} \\
\mathbf{m} \diamond \uparrow \frac{S\{\square(R, T)\}}{S(\square R, \square T)} & \mathbf{m} \square \uparrow \frac{S\{\bullet(R, T)\}}{S(\bullet R, \bullet T)} & \mathbf{m} \uparrow \frac{S\{\square(R, T)\}}{S(\boldsymbol{\square} R, \boldsymbol{\square} T)} \\
\mathbf{m} \diamond \downarrow \frac{S[\diamond R, \diamond T]}{S\{\diamond[R, T]\}} & \mathbf{m} \boldsymbol{\downarrow} \frac{S[\square R, \boldsymbol{\square} T]}{S\{\square[R, T]\}} & \mathbf{m} \downarrow \frac{S[\bullet R, \bullet T]}{S\{\bullet[R, T]\}}
\end{array}
$$

The proof of the following lemma is similar to previous lemmata concerning the interaction and weakening rules: that is, by establishing a set of reductions of general (co-)contraction rules to smaller contractions, with the help of the (co-)medial rules. But first we need to show that the medial rules are sound.

Lemma 21

The medial rules $\mathbf{m}, \mathbf{m} \square \downarrow, \mathbf{m} \diamond \downarrow, \mathbf{m} \mathbf{\square}$ and $\mathbf{m} \diamond \downarrow$ are derivable in $\{\mathbf{c} \downarrow, \mathbf{w} \downarrow\}$. Dually, the co-medial rules are derivable in $\{\mathbf{c} \uparrow, \mathbf{w} \uparrow\}$. 
Proof. We show the derivations for the down-rules; the derivations for the up-rules can be obtained by dualizing the derivations of the down rules. The rule $\mathbf{m}$ is derived as below left where $\mathbf{w} \downarrow^{*}$ denotes several applications of the $\mathbf{w} \downarrow$ rule. The rule $\mathbf{m} \square \downarrow$ is derived as shown below right:

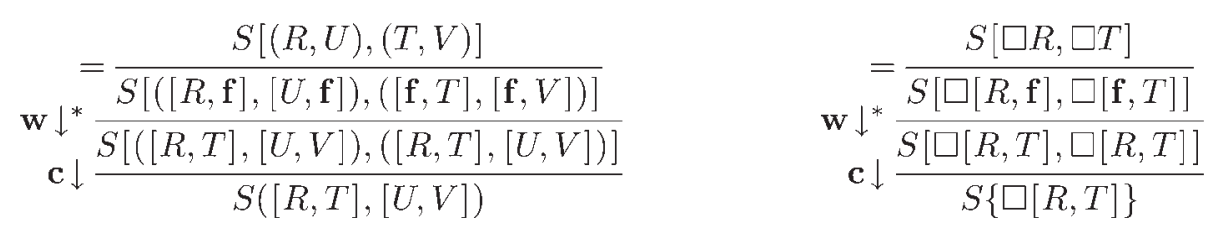

The derivations for $\mathbf{m} \diamond \downarrow, \mathbf{m} \mathbf{\square} \downarrow$ and $\mathbf{m} \diamond \downarrow$ are similar to the derivation of $\mathbf{m} \square \downarrow$ above, except that we replace $\square$ with, respectively, $\diamond, \mathbf{\square}$ and

LeMma 22

The contraction rule $\mathbf{c} \downarrow$ is derivable in $\{\mathbf{a c} \downarrow, \mathbf{m}, \mathbf{m} \square \downarrow, \mathbf{m} \diamond \downarrow, \mathbf{m} \mathbf{\square} \downarrow, \mathbf{m} \downarrow \downarrow\}$. Dually, the co-contraction rule $\mathbf{c} \uparrow$ is derivable in $\{\mathbf{a c} \uparrow, \mathbf{m}, \mathbf{m} \square \uparrow, \mathbf{m} \diamond \uparrow, \mathbf{m} \mathbf{\square} \uparrow, \mathbf{m} \diamond \uparrow\}$.

Proof. By induction on the size of the contracted structure. We apply the following reductions to instances of contraction, to reduce them to contraction on smaller size. The derivation of co-contraction is done dually.

$$
\begin{aligned}
& \mathbf{c} \downarrow \frac{S[[R, T],[R, T]]}{S[R, T]} \quad \mathbf{c} \downarrow \frac{S[(R, T),(R, T)]}{S(R, T)} \quad \mathbf{c} \downarrow \frac{S[\bigcirc R, \bigcirc R]}{S\{\bigcirc R\}} \\
& \Downarrow \quad \Downarrow \quad \Downarrow
\end{aligned}
$$

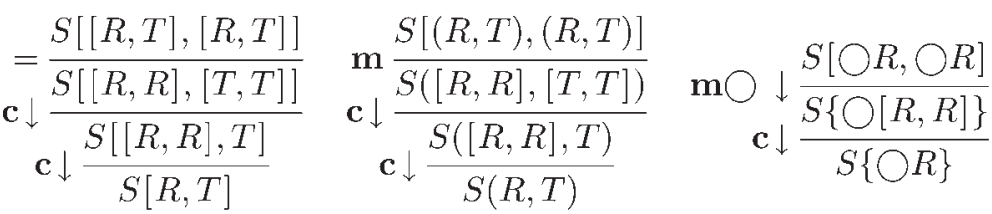

The reduction rules for structures with modalities follow the general pattern above, where $\bigcirc$ represents one of the modalities $\square, \diamond, \mathbf{\square}$ or $\diamond$. The concrete derivation for each of the cases $\square, \diamond, \boldsymbol{\square}$ and $\diamond$ is obtained by replacing $\bigcirc$ by the corresponding modality.

We are now ready to present a fully local CoS system for CMDL, which is obtained by reducing the non-local rules to their local counterparts according to the previous discussions.

DeFinition 23

System MSa is given in Figure 7. System SMSa is the symmetric closure of MSa.

THEOREM 24

Systems MS, MSa, SMS and SMSa are equivalent.

Proof. Straightforwardly from Theorem 13, Lemma 19, Lemma 20, Lemma 21 and Lemma 22.

\section{Capturing primitive extensions of CMDL}

In this section we consider extensions of CMDL with axiom schemes of the form $A \supset B$ where $A$ and $B$ are primitive formulae, that is, formulae that are formed using propositional variables 


$$
\begin{aligned}
& \text { ai } \downarrow \frac{S\{\mathrm{t}\}}{S[a, \bar{a}]} \quad \mathrm{s} \frac{S([R, T], U)}{S[R,(T, U)]} \quad \mathbf{m} \frac{S[(R, U),(T, V)]}{S([R, T],[U, V])} \\
& \text { ac } \downarrow \frac{S[a, a]}{S\{a\}} \quad \text { aw } \downarrow \frac{S\{\mathbf{f}\}}{S\{a\}} \quad \text { uwf } \downarrow \frac{S\{\mathbf{f}\}}{S\{\mathbf{f}\}} \quad \text { uwp } \downarrow \frac{S\{\mathbf{f}\}}{S\{\square \mathbf{f}\}} \\
& \mathbf{k f} \downarrow \frac{S\{\square[R, T]\}}{S[\diamond R, \square T]} \quad \mathbf{k p} \downarrow \frac{S\{\square[R, T]\}}{S[\bullet R, \square T]} \quad \mathbf{r p f} \downarrow \frac{S\{\square[\square R, T]\}}{S[R, \square T]} \quad \mathbf{r p p} \downarrow \frac{S\{[\square R, T]\}}{S[R, \mathbf{\square} T]}
\end{aligned}
$$

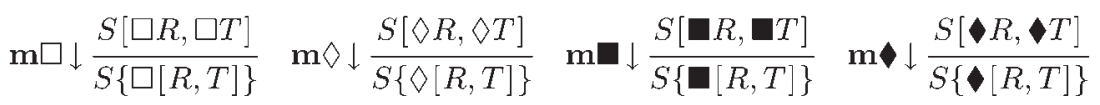

Figure 7. MSa: A local system for tense logic

and the connectives $T, \wedge, \vee, \diamond$ and $\diamond$. In CMDL, these axiom schemes are formalized as structural rules as follows [20].

Given a primitive axiom $A \supset B$, we transform $A$ and $B$ into the equivalent formulae shown below, using standard equivalences, and where all $C_{i}$ and $D_{j}$ are composed only from variables, $\mathrm{T}, \wedge, \diamond$ and

$$
A \equiv \bigvee_{i \leq m} C_{i} \quad B \equiv \bigvee_{j \leq n} D_{j}
$$

The formulae $C_{i}$ and $D_{j}$ are then translated to structures using the following function [20]:

$$
\begin{aligned}
\sigma(T) & =\mathbf{I} \\
\sigma(p) & =p \\
\sigma(P \wedge Q) & =\sigma(P) \circ \sigma(Q) \\
\sigma(\diamond P) & =* \bullet * \sigma(P) \\
\sigma(\diamond P) & =\bullet \sigma(P)
\end{aligned}
$$

The axiom $A \supset B$ is then turned into structural rules of the form shown below, one for each $k \leq m$ :

$$
\frac{\sigma\left(D_{1}\right) \vdash Y \quad \cdots \quad \sigma\left(D_{n}\right) \vdash Y}{\sigma\left(C_{k}\right) \vdash Y}
$$

It can be shown that $\tau_{1}\left(\sigma\left(C_{i}\right)\right)=C_{i}$ and $\tau_{1}\left(\sigma\left(D_{j}\right)\right)=D_{j}$. For each primitive axiom $F$, we denote with $d(F)$ the display rules obtained from $F$ by following the above transformations. We overload the notation for sets of axioms, i.e. given a set of primitive axioms $A x$, the notation $d(A x)$ denotes the set of display rules obtained from each axiom in $A x$.

To simulate the display rules $d(A \supset B)$ in $\operatorname{CoS}$, we employ a similar transformation from axioms to structural rules. Since our $\operatorname{CoS}$ rules are restricted to rules under positive context only, we shall work with the contrapositive forms of the axioms, and care has to be taken in handling negations in the contrapositive forms. 
Let $A^{\prime}$ be the formula scheme $\bar{A}$ (i.e. the negation normal form of $\neg A$ ) but with the negations on the schematic variables removed. Similarly, define $B^{\prime}, C_{i}^{\prime}$ and $D_{j}^{\prime}$ from $\bar{B}, \bar{C}_{i}$ and $\bar{D}_{j}$, respectively. The axiom $A \supset B$ is translated into the calculus of structures as below, one for each $k \leq m$ :

$$
\frac{S\left(\underline{D_{1}^{\prime}}, \ldots, \underline{D_{n_{\mathrm{s}}}^{\prime}}\right)}{S\left\{{\underline{C_{k}}}_{\mathrm{s}}^{\prime}\right\}}
$$

Note that in the CoS system SMS we restrict attention to structures in negation normal form. Since schematic variables can be instantiated with arbitrary structures, applying the rules with negations on the schematic variables will produce structures which are not in negation normal form. This is why we need to remove the negations on the schematic variables.

We use the notation $\rho(F)$ to denote a CoS rule obtained from the axiom $F$, and $\rho(A x)$ the set of $\mathrm{CoS}$ rules obtained from the set of axioms $A x$.

To show the soundness of the $\mathrm{CoS}$ rule, first observe that the following equivalences hold:

$$
\bar{A} \equiv \bigwedge_{i \leq m} \bar{C}_{i} \quad \bar{B} \equiv \bigwedge_{j \leq n} \bar{D}_{j}
$$

This can be shown to follow from the equivalences in (1) by taking their contrapositive forms. We note that since primitive formulae do not have negations, the negation normal forms of their negations will have all their variables negated. Now if we instantiate every variable $x$ in the above equivalences with its negation $\neg x$, then the resulting formulae will have all the variables double-negated, hence are equivalent to the following formulae which are both negation free:

$$
A^{\prime} \equiv \bigwedge_{i \leq m} C_{i}^{\prime} \quad B^{\prime} \equiv \bigwedge_{j \leq n} D_{j}^{\prime}
$$

With the same observations, we conclude that $B^{\prime} \supset A^{\prime}$ follows from $A \supset B$. The soundness of the rule then follows from a series of cuts on the following lemmata:
1. $\bigwedge_{j \leq n} D_{j}^{\prime} \supset B^{\prime}$
2. $B^{\prime} \supset A^{\prime}$
3. $A^{\prime} \supset \bigwedge_{i \leq m} C_{i}^{\prime}$
4. $\bigwedge_{i \leq m} C_{i}^{\prime} \supset C_{k}^{\prime}$.

\section{THEOREM 25}

Let $A x$ be a set of primitive axioms and let $R$ be a CoS structure. Then the following statements are equivalent.

(1) $R$ is provable in $\mathbf{S M S} \cup \rho(A x)$.

(2) $\underline{R}_{\mathrm{F}}$ is provable in CMDL $\cup d(A x)$.

(3) $\underline{R}_{\mathrm{F}}$ is cut-free provable in CMDL $\cup d(A x)$.

(4) $R$ is provable in MS $\cup \rho(A x)$. 
Proof. The proof is similar in most cases to the proof of Theorem 13 . We also rely on the cutelimination of CMDL extended with $d(A x)$ [20]. For the cases with primitive rules, it is obvious from the discussions above that the rule $d(F)$ corresponds to $\rho(F)$ for each primitive axiom $F \in A x$.

The curious reader may be wondering about the fifteen normal modal logics obtained by extending $K$ with the axioms $D, T, 4,5$ and $B$. That is, what happens if we add the five corresponding primitive axioms to our $\mathrm{CoS}$ calculi, but restrict ourselves to the modal language only? Using a semantic argument due to Wansing, it is easy to show that doing so gives the corresponding modal logic exactly. Thus, in particular, we also have cut-free CoS calculi for all of these fifteen normal modal logics. However, note that these calculi are extensions of MS, hence, they contain the residuation rules $\mathbf{r p p} \downarrow$ and $\mathbf{r p f} \downarrow$. Therefore, in a derivation of a modal formula, there could be occurrences of tense formulae in the derivation. ${ }^{1}$ In this sense, the extensions of MS with modal axioms, although capturing exactly the corresponding modal logics, are not pure. We would like to show that the residuation rules are admissible for extensions of MS with modal axioms, when restricted to the modal language. Whether this is achievable in general is still an open question. However, in the case of S5, it is easy to find pure, complete and cut-free CoS calculi because of symmetry, as shown in the next section.

\section{S5 as a primitive extension of CMDL}

We now show several examples of primitive extensions of CMDL which correspond to S5. Note that since CMDL is richer than normal modal logic, we need to restrict the extended systems to their modal fragments and show that they are sound and complete with respect to the usual formulations of $\mathbf{S 5}$ in Hilbert systems. The traditional formulations of axioms of S5 may not be primitive, we therefore need to find the corresponding primitive axioms in CMDL. We choose the modal-tense formulations that are most straightforward, from a semantical point of view, and then refine them to pure modal axioms. At the end of this section we will show that all these formulations in the calculus of structures are equivalent to a Hilbert system for $\mathbf{S 5}$.

\section{$8.1 \mathbf{S 5}=\mathbf{K T} \mathbf{4 B}$}

We consider the axiomatization of $\mathbf{S 5}$ which extends the basic normal modal logic $\mathbf{K}$ with the axioms $T, 4$ and $B$ shown as follows:

$$
T: P \supset \diamond P \quad 4: \diamond \diamond P \supset \diamond P \quad B: P \supset \square \diamond P
$$

Notice that the axiom $B$ is not primitive. An equivalent primitive axiom in tense logic which captures symmetric frames is

$$
B^{\prime}: \diamond P \equiv \diamond P
$$

\footnotetext{
${ }^{1}$ In display calculi, the tense connectives are disguised as structural connectives, giving the impression of a purely modal derivation.
} 
That is, a world $w$ makes $\diamond P$ true iff there exists some $v \in W$ with $w R v$ and $v$ makes $P$ true. But if $R$ is symmetric, then $w R v$ iff $v R w$. Hence $w$ makes $\diamond P$ true iff it makes $\diamond P$ true.

The axioms $T, 4$ and $B^{\prime}$ are translated to the following rules in $\mathrm{CoS}$ recalling that in translating the rules, we take the contrapositive forms of the axioms:

$$
\mathbf{t} \downarrow \frac{S\{\square R\}}{S\{R\}} \quad 4_{a} \downarrow \frac{S\{\square R\}}{S\{\square \square R\}} \quad \mathbf{b}_{1} \downarrow \frac{S\{\square R\}}{S\{\square R\}} \quad \mathbf{b}_{2} \downarrow \frac{S\{\square R\}}{S\{\square R\}}
$$

All these rules have their corresponding up-rules obtained by taking their contrapositive forms. As usual, we refer to the dual rules with the same names but with the arrows reversed.

By extending MS with the above rules, we are now able to prove the axioms $B$ and 5: $\diamond \square P \supset \square P$ as follows:

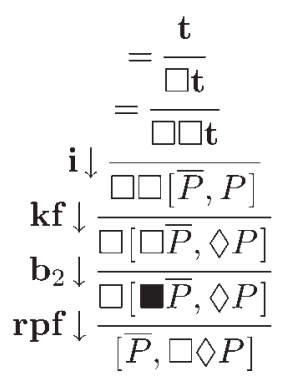

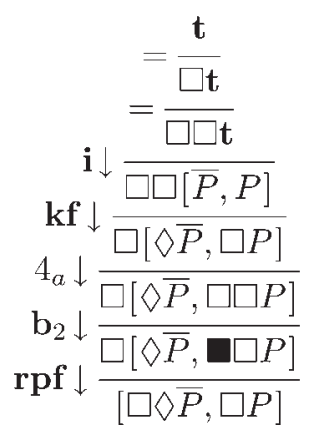

Notice that the derivations make use of non-trivial interactions between the tense fragment and the modal fragment of MS, i.e. via the use of the residuation rules $\mathbf{r p f} \downarrow$ and the $\mathbf{b}_{2} \downarrow$ rule.

However, the only occurrences of these rules seem to be needed when they appear in succession. This suggested the bigger-step rule $\mathbf{r b} \downarrow$ shown below left which combines the two and which is purely modal. The corresponding primitive axiom $B^{\prime \prime}$ for this rule is shown below right:

$$
\mathbf{r b \downarrow} \frac{S\{\square[\square R, T]\}}{S[R, \square T]} \quad B^{\prime \prime}: R \wedge \diamond T \supset \diamond(\diamond R \wedge T) .
$$

In the following we show that this alternative formulation $B^{\prime \prime}$ is indeed equivalent to $B$. More precisely, we show that the system obtained by extending MS with the axioms $T, 4$ and $B^{\prime \prime}$ captures $\mathbf{S 5}$.

DEFINITION 26

System MS $t 4 b$ is MS $\cup\left\{t \downarrow, 4_{a} \downarrow, \mathbf{r b} \downarrow\right\}$. System $\mathbf{S M S} t 4 b$ is the symmetric closure of MS $t 4 b$.

System SMS $t 4 b$ enjoys cut-elimination, as a consequence of Theorem 25.

THEOREM 27

System SMS $t 4 b$ and system MS $t 4 b$ are equivalent.

We now show that MS $t 4 b$ is also conservative over its modal fragment. 


$$
\begin{array}{ccc}
\mathbf{i} \downarrow \frac{S\{\mathbf{t}\}}{S[R, \bar{R}]} & \mathbf{s} \frac{S([R, T], U)}{S[R,(T, U)]} \quad \mathbf{c} \downarrow \frac{S[R, R]}{S\{R\}} \quad \mathbf{w} \downarrow \frac{S\{\mathbf{f}\}}{S\{R\}} \\
\mathbf{k f} \downarrow \frac{S\{\square[R, T]\}}{S[\diamond R, \square T]} & \mathbf{t} \downarrow \frac{S\{\square R\}}{S\{R\}} \quad 4_{a} \downarrow \frac{S\{\square R\}}{S\{\square \square R\}} \quad \mathbf{r b} \downarrow \frac{S\{\square[\square R, T]\}}{S[R, \square T]}
\end{array}
$$

Figure 8. System S5a, a modal subsystem of MS $t 4 b$

\section{DeFinItion 28}

System S5a is a subsystem of MSt4b, given by the rules in Figure 8. System SS5a is the symmetric closure of $\mathbf{S} 5 \mathbf{a}$.

DeFinItion 29

Let $R$ be a CoS structure. We denote with $\underline{R}_{\mathrm{M}}$ the CoS structure obtained from $R$ by changing every occurrence of $\boldsymbol{\square}$ in $R$ to $\square$ and every occurrence of $\downarrow$ to $\diamond$. The function ${ }_{-\mathrm{M}}$ is extended to $\mathrm{CoS}$ structure contexts by having $\underline{\{\}}_{\mathrm{M}}=\{\}$.

LemMa 30

If $\vdash_{\mathrm{MS} 4 t b} R$ then $\vdash_{\mathrm{S} 5 \mathrm{a}} \underline{R}_{\mathrm{M}}$.

Proof. Let $\Pi$ be a proof of $R$ in MS $4 t b$. We construct a proof $\Pi^{\prime}$ of $\underline{R}_{\mathrm{M}}$ in S5a by simply replacing instances of $\mathbf{k p} \downarrow$ in $\Pi$ with $\mathbf{k f} \downarrow$, and instances of $\mathbf{r p f} \downarrow$ and $\mathbf{r p p} \downarrow$ in $\Pi$ with $\mathbf{r b} \downarrow$.

THEOREM 31

Let $R$ be a modal CoS structure. Then the following statements are equivalent.
(1) $R$ is provable in $\mathbf{S M S} t 4 b$.
(2) $R$ is provable in $\mathbf{M S} t 4 b$.
(3) $R$ is provable in $\mathbf{S} 5 \mathbf{a}$.
(4) $R$ is provable in $\mathbf{S S} 5$ a.

Proof. This follows straightforwardly from Theorem 27 and Lemma 30.

\section{$8.2 S 5=K T 5$}

We now consider the axiomatizations of $\mathbf{S 5}$ using the axioms $K, T$ and 5. A simple primitive formulation of 5 in tense logic is:

$$
5^{\prime}: \diamond \diamond P \supset \diamond P .
$$

To see this, suppose that some world $x$ makes $\diamond \diamond P$ true. Thus $x$ has a predecessor $y$ which has a successor $z$, and $z$ makes $P$ true. Thus $y$ has both $x$ and $z$ as successors. The euclidean condition corresponding to axiom 5 demands that $z$ is a successor of $x$. That is, $x$ must make $\diamond P$ true too.

By viewing the above scenario from $y$ instead of $x$, we find a primitive modal axiom for 5 as below left, which translates to the CoS rule shown below right:

$$
5^{\prime \prime}: \diamond P \wedge \diamond Q \supset \diamond(P \wedge \diamond Q) . \quad \quad \mathbf{r} 5 \downarrow \frac{S\{\square[R, \square T]\}}{S[\square R, \square T]}
$$

We will use this axiom $5^{\prime \prime}$ and its $\mathbf{r} 5 \downarrow$ rule in the following discussion. 


$$
\begin{gathered}
\mathbf{i} \downarrow \frac{S\{\mathbf{t}\}}{S[R, \bar{R}]} \quad \mathbf{s} \frac{S([R, T], U)}{S[R,(T, U)]} \quad \mathbf{c} \downarrow \frac{S[R, R]}{S\{R\}} \quad \mathbf{w} \downarrow \frac{S\{\mathbf{f}\}}{S\{R\}} \\
\mathbf{k f} \downarrow \frac{S\{\square[R, T]\}}{S[\diamond R, \square T]} \quad \mathbf{t} \downarrow \frac{S\{\square R\}}{S\{R\}} \quad \mathbf{r} 5 \downarrow \frac{S\{\square[R, \square T]\}}{S[\square R, \square T]}
\end{gathered}
$$

Figure 9. System S5b: a modal subsystem of MS $t 5$

DEFINITION 32

System MS $t 5$ is MS $\cup\{\mathbf{t} \downarrow, \mathbf{r} 5 \downarrow\}$. System $\mathbf{S M S} t 5$ is the symmetric closure of MSt5.

THEOREM 33

Systems SMS $t 5$ and MS $t 5$ are equivalent.

Definition 34

System $\mathbf{S} 5 \mathbf{b}$, whose rules are given in Figure 9, is a modal subsystem of MSt5. System $\mathbf{S S} 5 \mathbf{b}$ is the symmetric closure of $\mathbf{S} 5 \mathbf{b}$.

LEMMA 35

If $R$ is provable in $\mathbf{M S} t 5$ then $\underline{R}_{\mathrm{M}}$ is provable in $\mathbf{S} 5 \mathbf{b}$.

Proof. The proof is similar to the proof of Lemma 30. The non-trivial cases are those concerning the residuation rules. We show here a derivation in $\mathbf{S} 5 \mathbf{b}$ of the 'M-image' of the residuation rule $\mathbf{r p f} \downarrow$ :

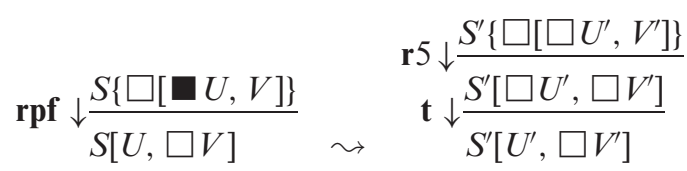

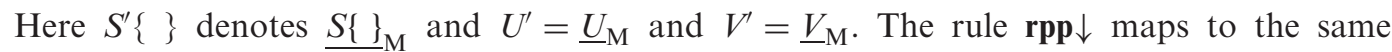
derivation.

The following theorem is a simple corollary of Lemma 35 and Theorem 33.

THEOREM 36

Let $R$ be a modal CoS structure. Then the following statements are equivalent.
(1) $R$ is provable in $\mathbf{S M S} t 5$.
(2) $R$ is provable in $\mathbf{M S} t 5$.
(3) $R$ is provable in $\mathbf{S} 5 \mathbf{b}$.
(4) $R$ is provable in $\mathbf{S S} 5 \mathbf{b}$.

\section{$8.3 S 5=K T 45$}

We now consider the formulation of $\mathbf{S 5}$ using the axioms $K, T, 4$ and 5 . In this section we review the existing system for KT45 in CoS due to Stouppa and Stewart [25, 26], which has been shown to be sound and complete with respect to Hilbert's system for KT45 and to have cut elimination. The purpose of this section is to show that our formulations of $\mathbf{S 5}$ in the previous sections, i.e. $\mathbf{S} 5 \mathbf{a}$ and $\mathbf{S} 5 \mathbf{b}$ are equivalent to their system. 


$$
\begin{array}{cccc}
\mathrm{i} \downarrow \frac{S\{\mathbf{t}\}}{S[R, \bar{R}]} & \mathrm{s} \frac{S([R, T], U)}{S[R,(T, U)]} & \mathbf{c} \downarrow \frac{S[R, R]}{S\{R\}} & \mathbf{w} \downarrow \frac{S\{\mathbf{f}\}}{S\{R\}} \\
\mathbf{k f} \downarrow \frac{S\{\square[R, T]\}}{S[\diamond R, \square T]} & \mathbf{t} \downarrow \frac{S\{\square R\}}{S\{R\}} & 4_{c} \downarrow \frac{S\{\diamond \diamond R\}}{S\{\diamond R\}} & 5_{c} \downarrow \frac{S\{\diamond \square R\}}{S\{\square R\}}
\end{array}
$$

Figure 10. System S5c of Stouppa and Stewart [27]

DEFINITION 37

System $\mathbf{S 5} \mathbf{c}$ is given in Figure 10. System $\mathbf{S S} 5 \mathbf{c}$ is the symmetric closure of $\mathbf{S 5 c}$.

THEOREM 38

System S5c and SS5c are equivalent [27].

THEOREM 39

Let $R$ be a modal CoS structure. Then the following statements are equivalent.
(1) $R$ is provable in $\mathbf{S} 5 \mathbf{a}$.
(2) $R$ is provable in $\mathbf{S} 5 \mathbf{b}$.
(3) $R$ is provable in $\mathbf{S} 5 \mathbf{c}$.

PROOF.

$1 \Rightarrow 2$ : We show that the rules of $\mathbf{S} 5 \mathbf{a}$ are all derivable in $\mathbf{S S} 5 \mathbf{b}$, and therefore every proof in

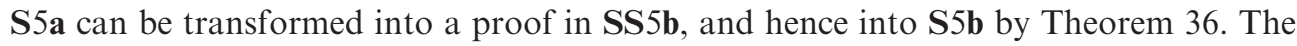
only cases to consider are the $4_{a} \downarrow$ and $\mathbf{r b} \downarrow$ rules:

$$
\begin{aligned}
& =\frac{S\{\square T\}}{S(\square \square \mathrm{t}, \square T)} \\
& \mathrm{i} \downarrow \overline{S(\square \square[\square T, \diamond \bar{T}], \square T)}
\end{aligned}
$$

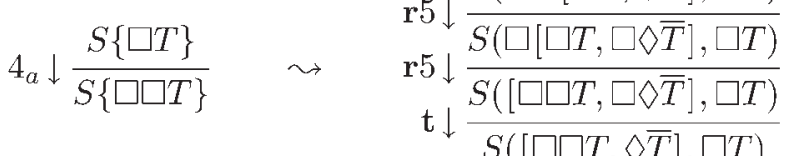

$$
\begin{aligned}
& \mathrm{t} \downarrow \frac{S([\square \square T, \square \diamond \bar{T}], \square T)}{S([\square \square T, \diamond \bar{T}], \square T)} \\
& \mathbf{r b \downarrow} \frac{S\{\square[\square U, V]\}}{S[U, \square V]} \leadsto \quad \leadsto \quad \mathrm{r} 5 \downarrow \frac{S\{\square[\square U, V]\}}{\mathrm{t} \downarrow} \frac{S[\square U, \square V]}{S[U, \square V]}
\end{aligned}
$$

$2 \Rightarrow 3$ : We show that every rule of $\mathbf{S} 5 \mathbf{b}$ is derivable in $\mathbf{S} 5 \mathbf{c}$. The only case to consider is $\mathbf{r} 5 \downarrow$ :

$$
\mathbf{r} 5 \downarrow \frac{S\{\square[U, \square V]\}}{S[\square U, \square V]} \quad \leadsto \quad \mathrm{kf} \downarrow \frac{S\{\square[U, \square V]\}}{S[\square U, \diamond \square V]}
$$


$3 \Rightarrow 1$ : We show that every rule in $\mathbf{S 5 c}$ is derivable in $\mathbf{S S} 5 \mathbf{a}$, and as in the first case $(1 \Rightarrow 2)$ we use the cut-elimination theorem for SS5a to get a proof in S5a. The non-trivial cases are the $4_{c} \downarrow$ and $5_{c} \downarrow$ rules

$$
4_{c} \downarrow \frac{S\{\diamond \diamond T\}}{S\{\diamond T\}} \quad 5_{c} \downarrow \frac{S\{\diamond \square U\}}{S\{\square U\}}
$$

Both can be derived by using cuts $(\mathbf{i} \downarrow)$ as in the first case $(1 \Rightarrow 2)$ : that is using the cuts $(\square \square \bar{T}, \diamond \diamond T)$ and $(\square \diamond \bar{U}, \diamond \square U)$. It is then enough to show that the following structures, which correspond to the axioms $\square A \supset \square \square A$ and $\diamond \square A \supset \square A$ defining the rules $4_{c} \downarrow$ and $5_{c} \downarrow$, are provable in $\mathbf{S} 5 \mathbf{a}$ :

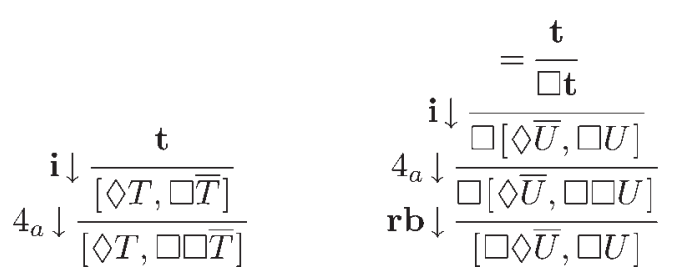

\section{Related and future work}

The closest existing work to ours is Stewart and Stouppa's formulation of S5, and some of its subsystems, in the calculus of structures [25]. We have seen their formulation of $\mathbf{S 5}$ as the system $\mathbf{S} 5 \mathbf{c}$ in Section 8.3. As in our case, their formulation of $\mathbf{S 5}$ relies on an external calculus for the cut-elimination result: in their case, it is the hypersequent formulation of S5 [1]. Stouppa [26] states that the formulation of S5 using only the axioms $T$ and 5 is an open problem, since their translation from hypersequents to the calculus of structures makes essential use of the 4 axiom. We have solved this problem by a detour through a primitive extension of CMDL, as detailed in Section 8.2, thereby justifying our claim of minimality.

Kashima [19] formalized tense logic and some of its extensions using two types of sequent calculi enriched with structural connectives ${ }^{F}\{$.$\} and { }^{P}\{$.$\} for the connectives \square$ and $\square$, respectively, similar to the single but overloaded structural connective $\bullet$ in display calculi. Kashima's first type of calculus contains explicit 'turn' rules to capture residuation as is done by the dp-rules for $\bullet$ in CMDL. His second type of calculus replaces these 'turn' rules by utilizing 'deep inference' to apply rules at an arbitrary depth in a sequent. Kashima presents extensions of tense logic with rules corresponding to transitivity, reflexivity, connectedness $(\forall x . \forall y . x R y \vee y R x \vee x=y)$ and totality $(\forall x . \forall y . x R y \vee y R x)$ of the underlying Kripke frames. It is known that a purely modal/tense Hilbert axiomatization cannot capture connectedness or totality, so no primitive extension of CMDL can handle these extensions. But it is also known that Hilbert axiomatizations of nominal tense logics can capture these conditions [4]. Thus it should be possible to obtain cut-free $\mathrm{CoS}$ calculi for these extensions by using primitive extensions of the display calculi for nominal tense logics given by Demri and Goré [10]. For the modal fragment, the second form of Kashima's sequent calculus was later rediscovered independently by Brünnler [5], under the name 'deep-sequent' calculus. Brünnler [5] presents the basic modal logics obtained by $K, T, D, 4,5$ and $B$, formalized using deep-sequent calculi. Brünnler states a conjecture that these axioms can be turned into 
structural rules in his calculus, from which one can obtain cut-free calculi for all extensions of classical logic with any subset of these axioms. Some of these structural rules, when translated properly into $\mathrm{CoS}$ rules, correspond to some of the rules for the axioms we show in Section 8, in particular, the rules $\mathbf{t} \downarrow$ and $\mathbf{r} 5 \downarrow$. Robert Hein [18] has independently come up with essentially the same set of rules for the axioms and the same conjecture for CoS, although as far as we know, the conjecture has not been proven.

We now list some avenues for further work:

Direct cut elimination for CoS: Our cut-elimination theorem for our modal and tense $\mathrm{CoS}$ calculi relies on that for display calculi. It still remains open to prove cut elimination directly in $\mathrm{CoS}$.

Conservativity: Prove the conservativity property for MS and its modal primitive extensions. More precisely, given a proof of a modal structure, we would like to be able to construct a proof of the same structure without using the residuation rules $\mathbf{r p f} \downarrow$ and $\mathbf{r p p} \downarrow$. This property, if proven, can be used to obtain a purely modal cut-free system for any modal primitive extensions of CMDL, among which are the various fragments obtained by extensions with $K, T, D, B, 4$ and 5 .

Fifteen Basic Modal Logics: Get a generic result for all the systems obtained by extensions with $K, T, D, B, 4$ and 5 . This is a consequence of the conservativity property given above. Improvements to Brünnler's Calculi: Brünnler's calculi [5] are not truly modular since it is not possible to just add and delete "axioms" at will as with Hilbert calculi. We would like to investigate whether our technique, via CMDL extensions, can lead to improvements to Brünnler's systems.

Kracht's Second Theorem: We now use the notation $x \triangleleft y$ for $R(x, y)$ and $x \triangleright y$ for $R(y, x)$. Kracht's first theorem [20] shows that the class of all properly displayable tense logics corresponds exactly to the class of first-order formulae of the form $\forall x(\forall)(\exists) M$ where $M$ is a matrix built from terms of the form $x=y$ and $x \triangleleft y$ with the help of $\wedge$ and $\vee$, and $(\forall)$ and $(\exists)$ are sequences of so-called 'two-way restricted quantifiers' $(\forall y \triangleright x),(\forall y \triangleleft x)$ and $(\exists y \triangleright x)$, $(\exists y \triangleleft x)$ in such a way that in every subformula $x \triangleleft y$ and $x=y$ in $M$, at least one of $x$ and $y$ is 'hereditarily universal', which means that it is not bound by an existential quantifier. Kracht claims in his second theorem that the class of properly displayed modal logics captures the same class, but this is known to be incorrect: the logic corresponding to the non-primitive axiom $\diamond \square p \supset \square \diamond \square p$ falls into the $\forall x(\forall)(\exists) M$ class, so the modal notion of primitivity for an axiom is too restrictive in forbidding occurrences of $\square$ outright. An open question is to find the exact first-order characterization of modal (rather than tense) display calculi.

\section{Conclusion}

We have shown that all tense logics properly displayable in the CMDL framework can be encoded in the calculus of structures. Using the cut-elimination result for CMDL, we have shown that these CoS calculi are cut free. Using Kracht's technique, we have also shown how to derive cut-free CoS calculi for arbitrary primitive extensions of CMDL.

We have applied our method to give cut-free formulations of several systems for S5 in CoS by extending CMDL with the modal primitive axioms for $\mathbf{S 5}$, translating the resulting cut-free systems into $\mathrm{CoS}$ calculi, and proving the required conservativity results for these systems. More precisely, in the last step, we show that any modal structure that is provable in the CoS 
system using the residuation rules $\mathbf{r p f} \downarrow$ and $\mathbf{r p p} \downarrow$, is also provable in the subsystem without these rules. Our technique relies on the symmetry of $R$ in $\mathbf{S 5}$ since we take a given proof of a structure with past-time modalities ( $\bullet$ and $\boldsymbol{\square}$ ), and find a proof for the same structure with these modalities replaced by the future-time modalities $(\diamond$ and $\square$ ). As far as we know, this is the first solution to the problem of finding a minimal and cut-free CoS calculus for S5.

Our work was motivated by the desire to improve the modularity of modal 'sequent' calculi. The calculus of structures seems a suitable framework for such a task for a number of reasons. First, the ability to translate axioms almost directly into $\mathrm{CoS}$ rules gives $\mathrm{CoS}$ calculi a modularity not (immediately) enjoyed by traditional modal sequent calculi. Second, unlike Hilbert calculi, CoS calculi enjoy a form of analyticity via the finite choice and locality properties of the rules that makes them more suitable for proof search, although the search itself may be highly non-deterministic. Third, $\operatorname{CoS}$ calculi are also more general than many non-standard sequent calculi, including intuitionistic calculi and hypersequent calculi, and as we demonstrate in this paper, are at least as general as (nominal) modal/tense display calculi.

The main drawback of modal CoS calculi, however, is that we know of no direct proofs of cut elimination for any modal $\mathrm{CoS}$ calculi since our cut-elimination proofs rely completely on the cut elimination results for modal display calculi.

\section{Acknowledgement}

We thank Kai Brünnler and the anonymous referees for their comments on an earlier version of the article. NICTA is funded by the Australian Government's Dept of Communications, Information Technology and the Arts and the Australian Research Council through Backing Australia's Ability and the ICT Centre of Excellence program.

\section{References}

[1] A. Avron. The method of hypersequents in the proof theory of propositional nonclassical logics. In Logic: from Foundations to Applications, European Logic Colloquium, pp. 1-32. Clarendon Press, Oxford, 1996.

[2] D. Basin, M. D’Agostino, D. M. Gabbay, S. Matthews, and L. Viganò, eds. Labelled Deduction. Vol. 17 of Applied Logic Series. Kluwer Academic Publishers, Dordrecht, 2000.

[3] N. D. Belnap. Display logic. Journal of Philosophical Logic, 11, 375-417, 1982.

[4] P. Blackburn. Nominal tense logic. Notre Dame Journal of Formal Logic, 34, 56-83, 1993.

[5] K. Brünnler. Deep sequent systems for modal logic. In Proceedings of the Advances in Modal Logic (AiML), 2006.

[6] K. Brüunnler. Deep Inference and Symmetry in Classical Proofs. PhD Thesis, TU Dresden, 2003.

[7] K. Brünnler and A. Tiu. A local system for classical logic. In LPAR 2001. Vol. 2250 of Lecture notes in Computer Science, R. Nieuwenhuis and A. Voronkov, eds, pp. 347-361. Springer, 2001.

[8] J. E. Dawson and R. Goré. Formalised cut admissibility for display logic. In Proceedings of the 15th International Conference on Theorem Proving in Higher Order Logics. V. A. Carreno, C. A. Munoz, and S. Tahar, eds, Vol. 2410 of Lecture Notes in Computer Science, pp. 131-147. Springer, 2002. 
[9] J. E. Dawson and R. Goré. A new machine-checked proof of strong-normalisation for display logic. In CATSO3: Proceedings of the 8th Australasian Theory Symposium, Adelaide, 2003. J. Harland, ed., Vol. 78 of ENTCS, pp. 16-35. Elsevier, 2003.

[10] S. Demri and R. Goré. Display calculi for nominal tense logics. Journal of Logic and Computation, 12, 993-1016, 2002.

[11] M. Fitting. Proof Methods for Modal and Intuitionistic Logics, Vol. 169 of Synthese Library. D. Reidel, Dordrecht, Holland, 1983.

[12] G. Gentzen. Untersuchungen über das logische schließen I and II. Mathematische Zeitschrift, 39, 176-210 and 405-431, 1935. English translation: Investigations into logical deduction. In The Collected Papers of Gerhard Gentzen, M. E. Szabo, ed., pp. 68-131. North-Holland, 1969.

[13] R. Goré. On the completeness of classical modal display logic. In Proof Theory of Modal Logic. H. Wansing, ed., Vol. 2 of Applied Logic, pp. 137-140, Kluwer, 1996.

[14] R. Goré. Gaggles, Gentzen, Galois: How to display your favourite substructural logic. Logic Journal of the Interest Group in Pure Applied Logic, 6, 669-694, 1998.

[15] R. Goré. Substructural logics on display. Logic Journal of the Interest Group in Pure Applied Logic, 6, 451-504, 1998.

[16] R. Goré. Chapter 6: Tableau methods for modal and temporal logics. In Handbook of Tableau Methods, M. D’Agostino, D. Gabbay, R. Hähnle, J. Posegga, eds, pp. 297-396. Kluwer Academic Publishers, 1999.

[17] A. Guglielmi. A system of interaction and structure. ACM Transactions on Computational Logic, 8, 1-64, 2007.

[18] R. Hein. Geometric Theories and Modal Logic in the Calculus of Structures. Master's Thesis, TU Dresden, 2005.

[19] R. Kashima. Cut-free sequent calculi for some tense logics. Studia Logica, 53, 119-135, 1994.

[20] M. Kracht. Power and weakness of the modal display calculus. In Proof Theory of Modal Logic, H. Wansing, ed., Kluwer, 1996.

[21] S. Negri. Proof analysis in modal logic. Journal of Philosophical Logic, 34, 507-544, 2005.

[22] G. Pottinger. Uniform, cut-free formulations of T, S4 and S5. Abstract in JSL, 48, 900-901, 1983.

[23] G. Restall. Displaying and deciding substructural logics I: logics with contraposition. Journal of Philosophical Logic, 27, 179-216, 1998.

[24] K. Schütte. Proof Theory, Springer-Verlag, 1977.

[25] C. Stewart and P. Stouppa. A systematic proof theory for several modal logics. In Advances in Modal Logic. R. Schmidt, I. Pratt-Hartmann, M. Reynolds and H. Wansing, eds, Vol. 5 of King's College Publications, pp. 309-333, 2005.

[26] P. Stouppa. The Design of Modal Proof Theories: The Case of S5. Master's Thesis, TU Dresden, 2004.

[27] P. Stouppa. A deep inference system for the modal logic S5. Studia Logica, in press.

[28] L. Straßburger. Linear Logic and Noncommutativity in the Calculus of Structures. PhD Thesis, Technische Universität Dresden, 2003.

[29] A. Tiu. A local system for intuitionistic logic. In LPAR 2006. M. Hermann and A. Voronkov, eds, Vol. 4246 of LNAI, pp. 242-256. Springer, 2006.

[30] A. Tiu. A system of interaction and structure II: The need for deep inference. Logical Methods in Computer Science, 2, 1-247, 2006. 
794 Classical Modal Display Logic

[31] L. Viganò. Labelled Non-Classical Logics. Kluwer Academic Publishers, Dordrecht, 2000.

[32] H. Wansing. Sequent calculi for normal modal propositional logics. Journal of Logic and Computation, 4, 125-142, 1994.

Received 4 May 2007 